\title{
Periocular Recognition: Analysis of Performance Degradation Factors
}

\author{
Chandrashekhar N. Padole and Hugo Proenca \\ IT: Instituto de Telecomunicacoes, \\ University of Beira Interior, \\ Covilha, Portugal \\ chandupadole@yahoo.com \& hugomcp@di.ubi.pt
}

\begin{abstract}
Among the available biometric traits such as face, iris and fingerprint, there is an active research being carried out in the direction of unconstrained biometrics. Periocular recognition has proved its effectiveness and is regarded as complementary to iris recognition. The main objectives of this paper are three-fold: 1) to announce the availability of periocular dataset, which has a variability in terms of scale change (due to camera-subject distance), pose variation and non-uniform illumination; 2) to investigate the performance of periocular recognition methods with the presence of various degradation factors; 3) propose a new initialization strategy for the definition of the periocular region-ofinterest (ROI), based on the geometric mean of eye corners. Our experiments confirm that performance can be consistently improved by this initialization method, when compared to the classical technique.
\end{abstract}

\section{Introduction}

Biometrics has been widely investigated and used effectively in several applications: authentication in highly restricted areas, attendance record in office premises, citizenship identification and verification and forensics. Different biometric traits such as face, iris, fingerprint and gait, exist to provide the flexibility to choose one or combine more than one modalities for recognition, as per the availability and feasibility associated with objectives of application. Due to easy availability and affordable cost of hardware, biometric has been a preferred choice even in personal devices like computers, PDA (Personal Digital Assistant) and mobile devices over a password based authentication. When it comes to usability, biometric recognition systems usually operate in constrained lighting scenarios and under rigid data acquisition protocols, although the development of non cooperative systems has been motivating numerous research efforts [19]. In this context, the main hurdles are the data quality decrease and its non-uniformity in terms of

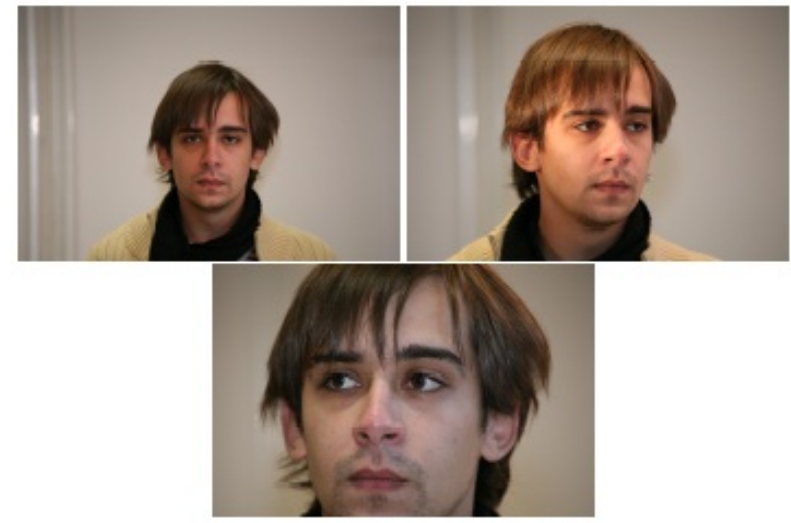

Figure 1. Examples of images of our periocular dataset, with variations in scale, lighting, pose and occlusions.

the traditional variation factors (translation, rotation, scale, pose and lighting changes).

Periocular biometrics refers to the recognition using information from the "facial region in the immediate vicinity of the eye [12]" has recently been identified as an extended form of iris recognition and has become an active research area for the research groups across the world. Despite of utilizing the iris region as a part, periocular biometrics can be preferred over the iris recognition for several reasons reported in existing literature. This could be enough reasoning to exploit the periocular biometrics in unconstrained requirements, among which the work of Park et al.[13] should be highlighted: they investigated the feasibility of periocular recognition in visible wavelength data acquired in relatively less constrained. In this paper, we intend to go beyond and measure the effect on periocular recognition performance with respect to each of the classical data variability factors also commonly called as "Covariates". For such, we built and released a new data set of periocular data, from where some examples can be seen in figure 1 . When compared to others, this data set has notoriously higher levels of heterogeneity and can constitute also 
a tool for the evaluation and development of more robust periocular recognition systems. Finally, we propose a new strategy to initialize the periocular ROI, based on the geometric mean of eye corners and that - as our experiments confirmed - can consistently improve performance when compared to the classical approach of initializing the ROI based on the location of the iris center.

The remainder of this paper is organized as follows: Section 2 briefly overviews existing literature for periocular biometrics. A brief description of the new database is given in Section 3. Section 4 elaborates pattern recognition descriptors that can be used for periocular recognition. The experimental result and the corresponding discussion will be presented in section 5. Finally, the paper will be concluded and several directions for further work pointed in section 6 .

\section{Related Work}

The first paper [13] of its kind, based on periocular biometrics has highlighted the benefits of biometrics recognition using periocular images especially when compared to iris recognition. Authors presented a feasibility study of periocular biometric recognition, when using local descriptors, LBP (Local Binary Pattern), HOG (Histogram of Oriented Gradients) and global descriptor SIFT (Shift Invariant Feature Transform) features. The performance result with these descriptors with and without eye brow was also reported by authors. Another interesting work [12], which is an extension of the first paper, has extensively presented results with various aspects of periocular recognition that include role of eyebrows, left or right eye information contribution, manual or automatic segmentation impacts, local and global feature's effectiveness, performance achieved by fusion with face and periocular biometrics, degradation due to partial occlusion over face images, effect of disguising the eyebrows, effect of masking the iris and eye region and analyzing the effect of pose variation and occlusion. The experiments carried in this paper used FRGC 2.0 database [10].

In a more recent paper [7], gender and ethnicity were identified using periocular images. Author used the LBP as feature extractor and Support Vector Machines (SVM) classifier for classification. Experiments were performed in the FRGC face dataset. A very important conclusion derived in this paper is that classification accuracy obtained by using the periocular images is comparable to that obtained by using entire face images for gender and ethnicity recognition. Another important paper [22] studied the effect of using fusion techniques on periocular and iris images for non-ideal images of the eye characterized by occluded irises, motion and spatial blur, poor contrast and illumination artifacts. The experimental results using MBGC database [14] shows that score level fusion can improve the recognition perfor- mance.

In another paper [20], author has applied genetic and evolutionary computations (GEC) to the problem of selecting optimized set of features. This work mainly includes the comparison of two GEC-based Type II feature extraction (GEFE) methods for periocular biometric recognition: a steady-state GA and an elitist Estimation of Distribution Algorithm (EDA). This methodology has enabled to select the features that not only improve the recognition accuracy but also to use minimum number of features in order to have less computational complexity. One of the interesting papers [2] has proposed a algorithm for periocular recognition in visible spectrum based on the fusion of global matcher, GIST, and circular linear binary pattern (CLBP) features. This paper also investigates the effect of capture distance on the performance of periocular biometrics using UBIRIS.v2 database [15]. In an attempt to analyze the texture of periocular samples, Miller et al.[8] have presented a performance evaluation of a local periocular texture based recognition approach. Their work specifically examines the effect of quality of samples on performance of recognition system.

However, it is essential to mention that the work presented in this paper is different from that in [8] in the sense that former investigates the effect of covariates present in actual acquired samples in terms of scale, pose, pigmentation level, gender, occlusion with uncontrolled illumination; while later uses few simulated degradation factors like blurring, resolution with controlled and uncontrolled lighting. In fact, performing experiments with actual covariate factors in periocular images introduced by image capturing framework itself, than simulated one, is the main objective of introducing UBIPr dataset that is used in our work.

\section{Database Description}

The most well known data sets to perform experiments on periocular recognition are the Multiple Biometrics Grand Challenge (MBGC) and Face Recognition Grand Challenge (FRGC). However, from our viewpoint, these data sets do not provide enough data variability: they have only minor variations in pose, few illumination diversity and absence of scale changes. Bharadwaj et al. [2] have used UBIRIS.v2 dataset, which has noisy images of iris. This dataset is created with the objective of being used in iris recognition research and some samples of these dataset don't include eyebrows and sometimes other parts of periocular region. This shortcoming makes inappropriate to use this dataset for periocular recognition despite of presence of various noise (degradation) factors. Another drawback of UBIRIS.v2 is that it doesn't include the pose information in its ground truth data.

According to the above observations, we built a new data set able to be used in periocular recognition experiments in non-controlled acquisition conditions and setups. 
Table 1. Image Acquisition and Dataset Specifications

\begin{tabular}{|l|l|}
\hline \multicolumn{2}{|c|}{ Image Acquisition \& Ground Truth } \\
\hline Camera & Conon EOS 5D \\
\hline Focal length & $400 \mathrm{~mm}$ \\
\hline Lens Aperture & F/6.4 - F/7 \\
\hline Color Representation & sRGB \\
\hline Shutter Speed & $1 / 197$ sec. \\
\hline F-Number & F/6.3 - F/7.1 \\
\hline Exposure Time & $1 / 200$ sec. \\
\hline ISO Speed & ISO-1600 \\
\hline Metering Mode & Pattern \\
\hline \multicolumn{2}{|c|}{ Periocular Image Specification } \\
\hline Camera Distance & Image Resolution \\
\hline $8 \mathrm{~m}$ & $501 \times 401$ pixels \\
\hline $7 \mathrm{~m} \quad$ Volunteers \\
\hline $6 \mathrm{~m}$ & $561 \times 541$ pixels \\
\hline $5 \mathrm{~m}$ & $651 \times 501$ pixels \\
\hline $4 \mathrm{~m}$ & $801 \times 651$ pixels \\
\hline Image Format & $1001 \times 801$ pixels \\
\hline \multicolumn{2}{|c|}{ bmp (Color) } \\
\hline $\begin{array}{l}\text { No of Subjects = 261 (with 104 in 2 } \\
\text { sessions), Periocular Regions =522 } \\
\text { Total Images= (261+104)x15x2 } \\
=10950\end{array}$ & $\begin{array}{l}\text { Male= } \\
\text { Female= 45.6\% }\end{array}$ \\
\hline
\end{tabular}

This data set will be made public and freely available at the web site [21]. We intended to embed on this dataset as much data variability factors as we could: data was acquired from highly different subject-camera distance, under distinct types and levels of illumination, poses and occlusions. Additionally, the manual annotation of dataset, including the ROI and location of essential landmarks, is made available on same website. The ground truth generation was observer centric which means it was performed with the process as classified as "manual annotation" type in [9]. The set of specifications used for the imaging framework is given in Table 1.

\section{Park et al. Periocular Method}

Park et al. [12] considered three types of features to assess the feasibility of periocular recognition. These features are described below briefly:

\subsection{HOG}

Among many, HOG is one of the local descriptors that has given promising performance in variety of problems of computer vision $[18,17]$. The image is decomposed into local regions and from each local region gradient orientation and its magnitude are calculated. In each bin of gradient orientation of histogram, corresponding magnitudes are accumulated for the local region. It is believed that HOG is robust to illumination variation for recognition problems [4].

\subsection{LBP}

After using linear binary pattern (LBP) first time for measuring the local image contrast [11], it has been applied in several pattern classification problems $[3,5]$. To calculate LBP, each pixel is assigned with a label by a type of binary pattern obtained in 3x3-neighborhood pixels by thresholding neighborhood pixel intensity with center pixel. The distribution of these binary patterns in local region is used as a feature representation, describing the nature of texture exist in that region.

\subsection{SIFT}

Lowe [6] has introduced the shift invariant feature transform to describe the image globally. Its invariance nature to rotation, scaling and translation has been successfully used in several applications to get improved performance over other features. SIFT features calculated as a difference of Gaussian (DoG) filtered images for two different scales for few numbers of octaves (down sampled versions of images). Key points are extracted where extrema of DoG is found.

\section{Experiments}

In order to perform the experiments over the new data set and to have experiments in line with main objectives of our research work, we divided them into three categories:

- Evaluation of descriptors with linear and non-linear fusion at match score level

- Comparison of performance obtained with two different types of references, eye corners proposed by us and iris center.

- Investigation of features for subsets of datasets, selected on the basis of pose variation, scale change, pigmentation level, with or without occlusion and gender

\subsection{Fusion at the Score Level}

One of the key points of periocular recognition performance is the way the scores generated by each of the three types of descriptors (HOG, LBP and SIFT) are fused. In this context, we tested two different techniques: linear and non-linear. For both cases, rectangular region of size $0.9 \mathrm{EW}$ $\mathrm{x} 1.2 \mathrm{EW}$ with its center being an iris center was cropped to select it as a periocular image, where EW is the eye width and it is the horizontal distance between nasal and temporal eye corner. This size was chosen to cover complete structure of periocular region across all the subjects. For HOG and LBP, this region was further divided in $6 \times 8$ blocks of grid as shown in figure 3 . HOG and LBP was then applied 
to each of the block with number of bins 8 and 32, respectively. Global descriptors SIFT was applied iteratively to each of periocular region until the number of descriptor locations reaches to less than or equal to 200 by using adaptive threshold. To calculate SIFT features, we used toolkit available publicly on [1]. These three features were fused using linear and non-linear fusion at match score level as described below:

\subsubsection{Linear Fusion}

Linear fusion [16] using the Logistic Regression can be achieved by assigning the weights to match score output of each feature classifier and combining them as given by equation 1 .

$$
S_{k}=\sum_{i=1}^{N} w_{i} r_{i, k}
$$

where $w_{i}$ is the weight assigned to output of $i^{\text {th }}$ classifier and $r_{i, k}$ is the match score of $i^{t h}$ classifier, where $\mathrm{i}=1, \ldots \ldots, \mathrm{N}$. These weights are determined by the Logistic Linear Regression. Here, we used three classifiers $(\mathrm{N}=3)$ based on HOG, LBP and SIFT features and least square method was used for fitting regression model.

\subsubsection{Non-linear Fusion}

Multi Layer Perceptrons (MLP) can be used to fuse the scores from more than one classifiers to realize non-linear fusion at match score level. The match scores obtained from each classifier are considered as input features for the MLP classifier. This neural network classifier then trained with genuine and impostor scores. The MLP used has parameters as given below:

- Nodes in Input, Hidden Layer, Output $\}=\{3,3,1\}$

- Activation functions $=\{$ sigmoid, sigmoid, linear $\}$

- Epochs and Performance Goal= 20000 and $10^{-3}$

The performance for linear and non-linear fusion of above mentioned descriptors are shown in figure 2. Due to superior data adaptive capability of neural network, the performance with non-linear fusion found to be slightly better than that with linear fusion. The experiments hereafter have used non-linear fusion to show the performance of periocular recognition.

\subsection{Effect of Reference Points : Iris Center vs. Eye Corners}

In pattern recognition, alignment of samples across intraclass and inter-class subjects, plays vital role, especially, when features are extracted as a local descriptors in spatial domain. This fact yields the importance in selecting the

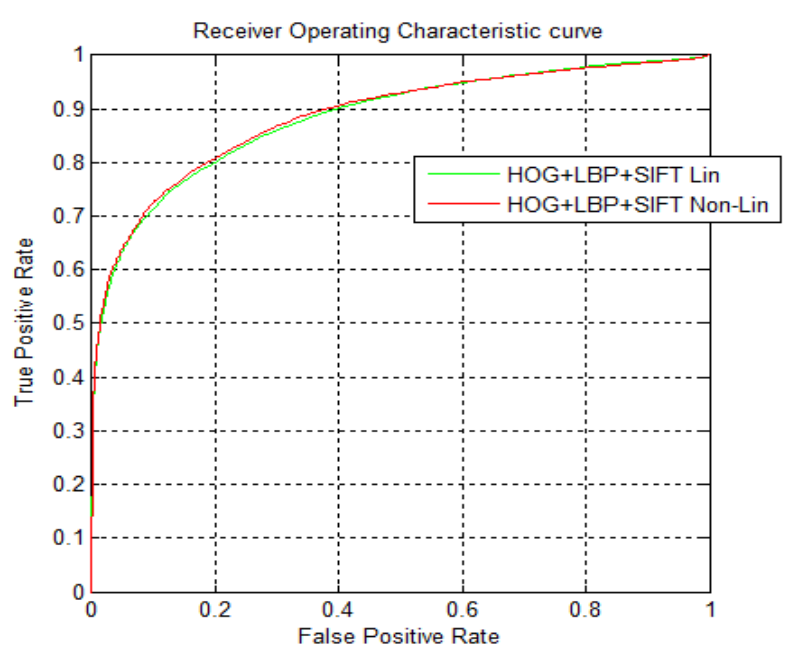

Figure 2. Recognition performance with linear and non-linear fusion of HOG, LBP and SIFT at match score level

reference points about which periocular region of interest (ROI) would be cropped for further processing. In [12], reference point has been used as iris center and being this point as a center, rectangular region of $3 \mathrm{Rx} 4 \mathrm{R}$ pixels was selected from the face images, where $\mathrm{R}$ is the radius of iris. The performance of periocular recognition is satisfactory with this approach in the case of frontal images acquired in controlled environment. However, iris being a moving entity of an eye can degrade the intra-class and inter-class alignment, if face or periocular samples are captured in unconstrained manner, where pose and gaze deviations would occur frequently. So, we propose a reference point to be determined from eye corners, nasal and temporal eye corner, to select the periocular region over an iris center, as shown in figure 2 , for following reasons:

- location of eye corners will have no deviations in structure of periocular region

- in case of closed eye, eye corners would still be detectable as opposed to the impossibility of detecting iris center

In addition to periocular region reference points, we also use eye width, which is the distance between nasal and temporal eye corners, as a reference for selecting size of periocular region instead of radius of iris, proposed in [12]. The reason for this is similar to those mentioned for choosing the eye corners as reference point. We hypothesize that eye center selected from two eye corners can give uniformity across the periocular regions of intra-class and inter-class subjects. The horizontal location of reference point is the midpoint of horizontal locations of nasal and temporal eye corner. Vertical location can be fixed with the vertical location of iris or average vertical location of eye corners. The effect of two 


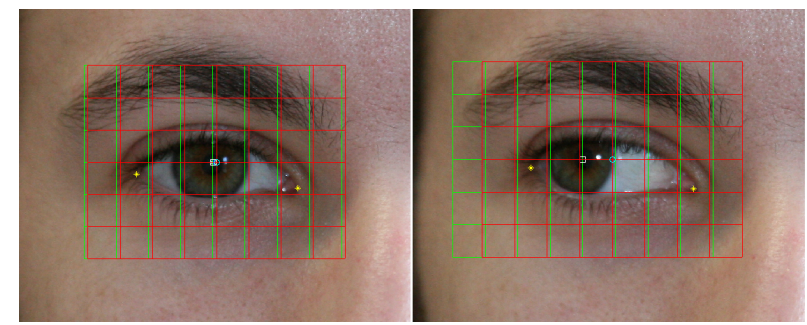

Figure 3. Effect of references as iris center (green grids) or the geometric mean of eye corners (red grids) in the definition of the ROI. Notice the higher correspondence between the red grids from frontal and deviated samples.

different types of reference points is illustrated in figure 3. It can be observed that, in case of frontal samples (Left), both reference points does not differ much and locate the periocular regions (green and red grid) in almost same vicinity and hence, they are overlapped. In samples of gaze (or pose) deviated (Right), difference in locating periocular regions by both reference points is clearly visible. With iris center, periocular region shifted much towards temporal side of the face due to shift in iris location. However, periocular region has maintained its location aligned over eye region in case of eye corner reference point. Moreover, the alignment preserved by periocular region in case of eye corners for frontal and deviated samples (red grids) is much more dominant as compared to that with iris center as reference point (green grids). We have tested experimentally the hypothesis of using eye corners as a reference points and eye width as a reference size for periocular region over a iris center and its radius. ROC curves for both references using non-linear fusion of features are shown in figure 4. It supports the use of eye corners as reference points to select the periocular region especially in case of unconstrained biometrics, where pose, gaze variations are bound to happen.

\subsection{Covariates}

In order to analyze the periocular recognition performance in different situations, we carried out the experiments using non-linear fusion of features at score level over the all possible types of subsets classified based on the covariates such as scale, pose, occlusion, pigmentation level and gender.

\subsubsection{Scale Wise Performance}

The ROC curves for periocular recognition of subset datasets of different scales are shown in figure 5. As periocular region size is determined proportional to the eye width, descriptors were scale invariant. However, due to presence of detailed information in high resolution, scale of $4 \mathrm{~m}$ (camera distance) subset has shown degradation in its performance. On the other hand, scale $7 \mathrm{~m}$ subset has desired

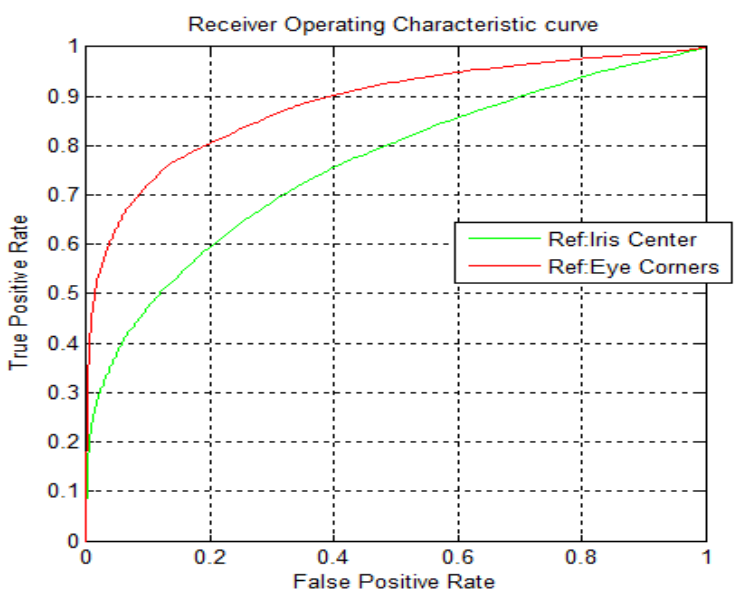

Figure 4. ROC curves for non-linear fusion of descriptors for reference points

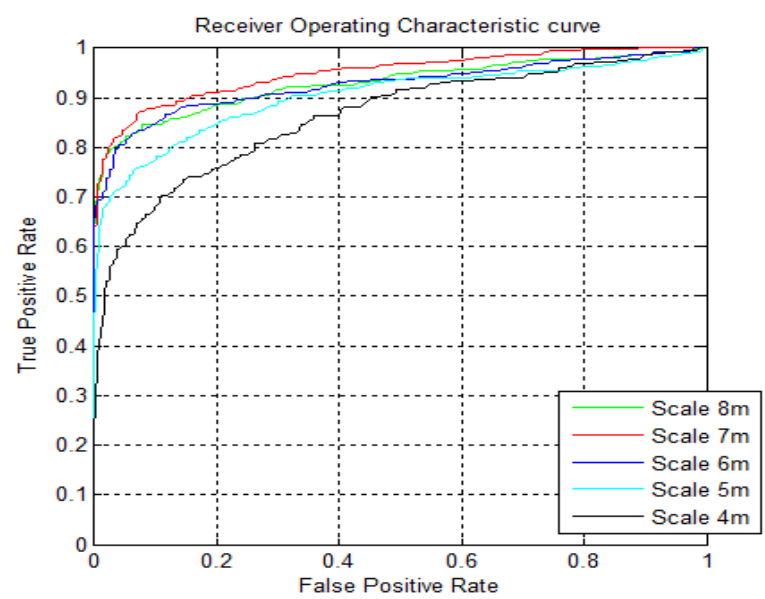

Figure 5. ROC curves for different scales

amount of detail in periocular samples and hence showing best performance. However, samples of scale $8 \mathrm{~m}$ subset contain short of information and scale $6 \mathrm{~m}$ subset samples include more than necessary information, thus, both subsets showing degraded performance as compared to the scale $7 \mathrm{~m}$ subset. This shows the performance of periocular recognition is dependent on the camera distance with which the sample was captured.

\subsubsection{Pose Wise Performance}

The performance results with subsets of various pose deviations samples are shown in figure 6 . It can be observed that more the deviations in pose of subjects, more degradation occurs in recognition performance. This degradation is due to the more misalignment and lesser information present in the one side of the periocular region image in pose deviated 


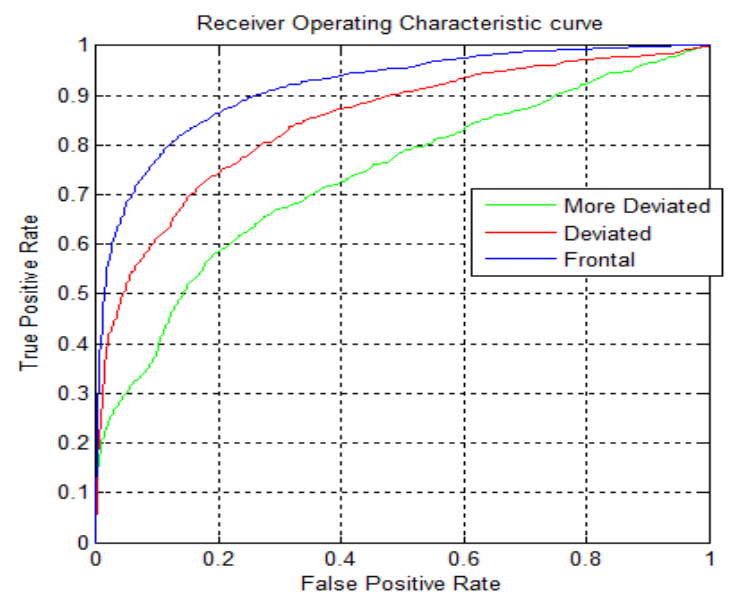

Figure 6. ROC curves for different poses

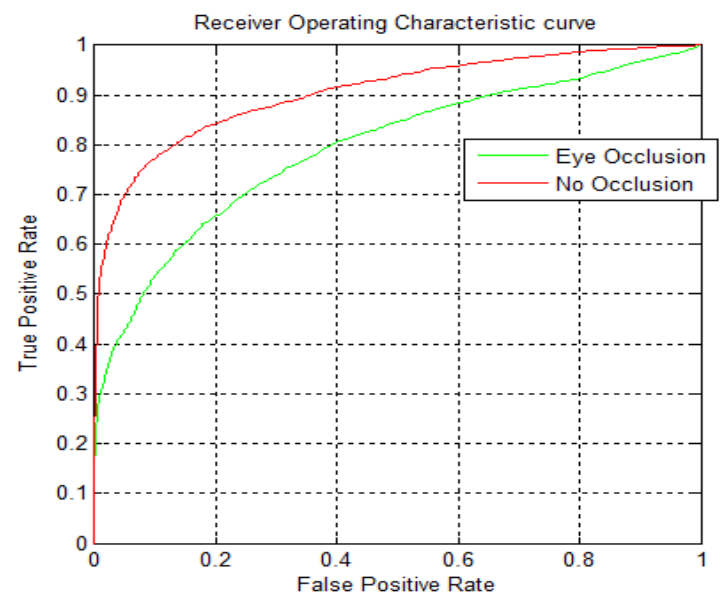

Figure 7. ROC curves with and without occlusion

sample. This fact confirms the need of having good overlapping across the intra-class and inter-class samples by using the techniques where pose variation can be compensated or corrected.

\subsubsection{With/without Occlusion Performance}

The results obtained from subsets of with occlusion and occlusion-free samples are plotted in figure 7 . Due to the occlusion, all the three types of descriptors get affected negatively. HOG and LBP fail to reproduce the local regions those comes under the occlusion. SIFT descriptor also fails in locating key point locations due to occluded region of the periocular image. This causes degradation in the recognition when periocular region is occluded.

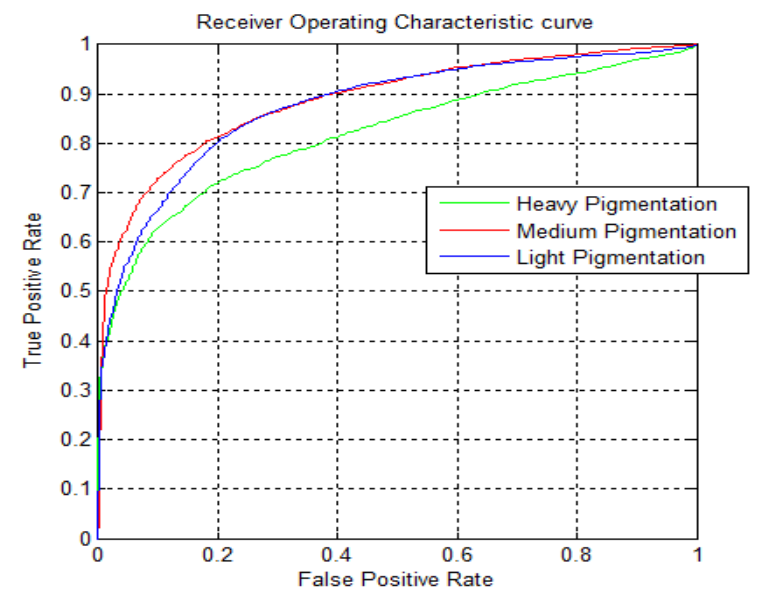

Figure 8. ROC curves for different pigmentation levels

\subsubsection{Pigmentation Level Performance}

Figure 8 shows the graphs plotted for the different levels of pigmentation. The pigmentation levels, light, medium and heavy, are decided as explained in [15]. This graph shows very little difference in the performance with light and medium pigmentation level samples at higher false positive rate. At lower false positive rate, where performance becomes crucial for biometric applications, periocular samples with medium pigmentation show better result as compared to the light pigmentation samples. However, performance is degraded in case of heavy pigmented irises due to low contrast that is frequently visible in iris patterns.

\subsubsection{Gender:Male/Female Performance}

Two different experiments were performed on male and female samples and ROC curves for them are shown in figure 9. It is interesting to see the biased capabilities of fused descriptors towards male and female subsets. This indicates the need of enhancing techniques to be used in case of female periocular sample. The degradation in performance of females subset may have happened due to possible makeup, eye brow coloring, use of glasses and presence of ear rings.

\section{Conclusion}

Growing research efforts have been put in the development of biometric recognition system that can operate in less constrained conditions and can reliably recognize individuals without requiring their active participation. In these type of systems, periocular recognition is believed to be vital and is expected to complement the iris as one of the preferable traits to be used in non-cooperative setups. In this paper we introduced a new data set with far more variation factors than that in the existing datasets. Also, we 


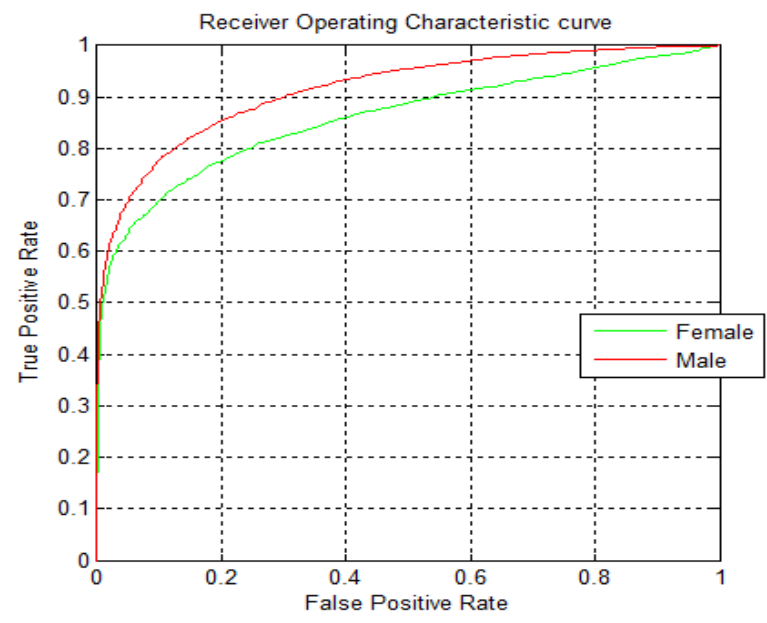

Figure 9. ROC curves for Gender

assessed the effect in performance with respect to each of the covariates type and proposed a new strategy to initialize the periocular ROI. This data initialization technique was observed to consistently outperform the classical one in all our experiments.

\section{Acknowledgments}

We acknowledge the financial support given by 'FCTFundacao para a Ciencia e Tecnologia' and 'FEDER' in the scope of the PTDC/EIA/103945/2008 'NECOVID: Negative Covert Biometric Recognition' and the support given by IT: Instituto de Telecomunicacoes in the scope of NOISYRIS research project .

\section{References}

[1] http://www.vlfeat.org/ vedaldi/code/sift.html.

[2] S. Bharadwaj, H. Bhatt, M. Vatsa, and R. Singh. Periocular biometrics: When iris recognition fails. In Fourth IEEE International Conference on Biometrics: Theory Applications and Systems (BTAS), 2010, pages 1-6, Sept. 2010.

[3] Y. Fang and Z. Wang. Improving LBP features for gender classification. In International Conference on Wavelet Analysis and Pattern Recognition, 2008. ICWAPR'08., volume 1, pages 373-377, Aug. 2008.

[4] L. Hu, W. Liu, B. Li, and W. Xing. Robust motion detection using histogram of oriented gradients for illumination variations. In 2nd International Conference on Industrial Mechatronics and Automation (ICIMA), 2010, volume 2, pages 443-447, May 2010.

[5] S. Junding, Z. Shisong, and W. Xiaosheng. Image retrieval based on an improved CS-LBP descriptor. In The 2nd IEEE International Conference on Information Management and Engineering (ICIME), 2010, pages 115-117, April 2010.

[6] D. G. Lowe. Distinctive image features from scale-invariant keypoints. International Journal of Computer Vision, 60:91110.
[7] J. Lyle, P. Miller, S. Pundlik, and D. Woodard. Soft biometric classification using periocular region features. In Fourth IEEE International Conference on Biometrics: Theory Applications and Systems (BTAS), 2010, pages 1-7, Sept. 2010.

[8] P. Miller, J. Lyle, S. Pundlik, and D. Woodard. Performance evaluation of local appearance based periocular recognition. In Fourth IEEE International Conference on Biometrics: Theory Applications and Systems (BTAS), 2010, pages 1-6, Sept. 2010.

[9] E. Murphy-Chutorian and M. Trivedi. Head pose estimation in computer vision: A survey. IEEE Transactions on Pattern Analysis and Machine Intelligence, , 31(4):607 -626, April 2009.

[10] NIST. Face recognition grand challenge database. http://www.frvt.org/FRGC/.

[11] T. Ojala, M. Pietikinen, and D. Harwood. A comparative study of texture measures with classification based on featured distributions. Pattern Recognition, 29(1):51-59, 1996.

[12] U. Park, R. Jillela, A. Ross, and A. Jain. Periocular biometrics in the visible spectrum. IEEE Transactions on Information Forensics and Security, 6(1):96-106, March 2011.

[13] U. Park, A. Ross, and A. Jain. Periocular biometrics in the visible spectrum: A feasibility study. In IEEE 3rd International Conference on Biometrics: Theory, Applications, and Systems, 2009. BTAS '09., pages 1-6, Sept. 2009.

[14] J. Philips. Multiple biometrics grand challenge. http://face.nist.gov/mbgc/.

[15] H. Proenca, S. Filipe, R. Santos, J. Oliveira, and L. Alexandre. The ubiris.v2: A database of visible wavelength iris images captured on-the-move and at-a-distance. IEEE Transactions on Pattern Analysis and Machine Intelligence, 32(8):1529-1535, Aug. 2010.

[16] A. Ross, K. Nandakumar, and A. K. Jain. Handbook of multibiometrics. New York: Springer-Verlag, 2006.

[17] P. Rybski, D. Huber, D. Morris, and R. Hoffman. Visual classification of coarse vehicle orientation using histogram of oriented gradients features. In IEEE Intelligent Vehicles Symposium (IV), 2010, pages 921-928, June 2010.

[18] A. Satpathy, X. Jiang, and H.-L. Eng. Extended histogram of gradients feature for human detection. In 17th IEEE International Conference on Image Processing (ICIP), 2010, pages 3473-3476, Sept. 2010.

[19] M. Savvides, K. R. Jr., D. L. Woodard, and G. Dozier. Unconstrained biometric identification: Emerging technologies. Computer, 43:56-62, 2010.

[20] L. Simpson, G. Dozier, J. Adams, D. Woodard, P. Miller, K. Bryant, and G. Glenn. Genetic amp; evolutionary type ii feature extraction for periocular-based biometric recognition. In IEEE Congress on Evolutionary Computation (CEC), 2010, pages 1-4, July 2010.

[21] UBIPr. UBI periocular recognition dataset. http://socialab.di.ubi.pt/ ubipr.

[22] D. Woodard, S. Pundlik, P. Miller, R. Jillela, and A. Ross. On the fusion of periocular and iris biometrics in non-ideal imagery. In 20th International Conference on Pattern Recognition (ICPR), 2010, pages 201-204, Aug. 2010. 